\title{
Loss of ER $\alpha$ and FOXA1 expression in a progression model of luminal type breast cancer: Insights from PyMT transgenic mouse model
}

\author{
KASI McCUNE $^{1 *}$, RUTIKA MEHTA ${ }^{2 *}$, MANGESH A. THORAT ${ }^{2}$, \\ SUNIL BADVE $^{2}$ and HARIKRISHNA NAKSHATRI ${ }^{1,3^{*}}$ \\ Departments of ${ }^{1}$ Surgery, ${ }^{2}$ Pathology and Laboratory Medicine, ${ }^{3}$ Biochemistry and \\ Molecular Biology, Indiana University School of Medicine, Indianapolis, IN 46202, USA
}

Received May 19, 2010; Accepted July 12, 2010

DOI: $10.3892 /$ or_00000977

\begin{abstract}
The classification of breast cancer into multiple molecular subtypes has necessitated the need for biomarkers that can assess tumor progression and the effects of chemopreventive agents on specific breast cancer subtypes. The goal of this study was to identify biomarkers whose expression are altered along with estrogen receptor $\alpha(\mathrm{ER} \alpha)$ in the polyoma middle- $\mathrm{T}$ antigen (PyMT) transgenic model of breast cancer and to investigate the chemopreventive activity of phenethyl isothiocyanate (PEITC). The diet of PyMT female mice was fortified with PEITC $(8 \mathrm{mmol} / \mathrm{kg})$ and the mammary streak and/or gross tumors and metastases in lungs were subjected to immunohistochemical analyses for ER $\alpha$, FOXA1, and GATA-3. FOXA1 is associated with luminal type A cancers, while GATA-3 is a marker of luminal progenitor cell differentiation. In both control and PEITC-treated groups, there was a progressive loss of ER $\alpha$ and FOXA1 but persistence of GATA-3 expression indicating that the tumors retain luminal phenotype. Overall, the PyMT induced tumors exhibited the entire gamut of phenotypes from ER $\alpha+/ F O X A 1+/ G A T A-3+$ tumors in the early stage to ER $\alpha \pm$ /FOXA1-/GATA-3+ in the late stage. Thus, PyMT model serves as an excellent model for studying progression of luminal subtype tumors. PEITC treated animals had multiple small tumors, indicating delay in tumor progression. Although these tumors were histologically similar to those in controls, there was a lower expression of these biomarkers in normal luminal cells indicating delay in tumor initiation. In in vitro studies, PEITC depleted AldeFluor-positive putative stem/progenitor cells, which may partly be responsible for the delay in tumor initiation.
\end{abstract}

Correspondence to: Dr Harikrishna Nakshatri, C218E, Indiana University School of Medicine, 980 West Walnut St., Indianapolis, IN 46202, USA

E-mail: hnakshat@iupui.edu

*Contributed equally

Key words: estrogen receptor, GATA-3, FOXA1, breast cancer

\section{Introduction}

Molecular profiling studies have led to the classification of breast cancer into five intrinsic subtypes: luminal subtype A, luminal subtype B, HER2+, basal, and normal-like (1). Although luminal type A and luminal type B breast cancers both express estrogen receptor $\alpha(\mathrm{ER} \alpha)$, luminal type A breast cancers additionally express transcription factors GATA-3 and FOXA1 $(1,2)$. ER $\alpha$, FOXA1 and GATA-3 constitute a functional transcription factor network that determines estrogen dependence and response to antiestrogen treatment (3). On the other hand, luminal type B breast cancers express proliferation markers such as Ki67 and these cancers appear to be less dependent on estrogen and hence respond poorly to antiestrogen treatment (4). Although biologically plausible, it is not clear whether clinically observed antiestrogen resistance involves any progression from luminal type A to luminal type B breast cancers. Even if such a progression occurs, it is yet uncertain if it can be assessed through biomarker profiling studies. Using MCF-7 cell model system, we have demonstrated insulin-mediated progression of luminal A to luminal B phenotype, which was accompanied by reduced expression of FOXA1 and GATA-3 but not ER $\alpha$, enhanced growth factor signaling, and resistance to antiestrogen treatment (5). Animal models provide an effective opportunity to test this concept in the biological environment and translate to human breast cancer biology.

The polyoma middle $\mathrm{T}$ (PyMT) transgenic mouse has proven to be an effective in vivo model that illustrates the progression of breast cancer. Mammary tumors demonstrate a distinct progression from adenoma through low grade to high grade carcinoma and ultimately to systemic disease characterized by pulmonary metastasis (6). Polyoma middle $\mathrm{T}$ antigen is a membrane-attached protein and is a potent oncogene that is not expressed in human tissue. It targets signaling pathways often activated in human breast cancer including Src family kinases, ras oncogene, and PI3 kinase pathways. Its expression also results in up-regulation of the proliferation-related oncogene c-Myc, which is elevated in human breast cancer (6). Mammary hyperplasia in this mouse model can be detected by as early as four weeks. By 14 weeks, 
many of these lesions have not only progressed to carcinoma, but also have given rise to pulmonary metastasis. The progression of these tumors from early adenoma to advanced/ metastatic carcinoma has been correlated with changes in $\mathrm{ER} \alpha$ and progesterone receptor (PR) status (6). The tumors retain their ER $\alpha / \mathrm{PR}$ positivity through adenoma, which is similar to their expression in luminal type A tumors in humans. By the time these tumors progress to the stage of early carcinoma there is loss of PR expression subsequently followed by a decreased ER $\alpha$ expression in advanced/metastatic carcinoma. These expression patterns in the later stage of tumors are similar to that observed in human luminal type B tumors. If this model represents the progression of luminal type A to luminal type B tumors, then biomarkers like ER $\alpha$, FOXA1, and GATA-3 could be used to study this progression. Moreover, the effectiveness of chemopreventive agents in halting this progression could be studied through the use of these markers.

Phenethyl isothiocyanate (PEITC) is a compound that is derived from vegetables of the genus Brassica (7) and has demonstrable chemopreventive properties in rat esophagus, liver, and bladder cancers (8). It has several proposed mechanisms of action including induction of reactive oxygen species (ROS) secondary to disruption of the glutathione antioxidant system resulting in mitochondrial damage, inactivation of redox-sensitive molecules, and massive cell death (9).

This study endeavors to investigate two specific aims. The first was to further explore the PyMT transgenic mice as effective models of progression of invasive breast cancer by analyzing the expression of biomarkers that are characteristic of predominantly ER $\alpha$-positive luminal type A tumors, specifically ER $\alpha$, FOXA1, and GATA-3. Loss of FOXA1 expression has been shown to correlate with both decreased response to endocrine therapy as well as reduced survival in patients with luminal type A breast tumors $(2,10)$. Additionally, there is evidence to suggest a correlation between loss of GATA-3 expression with poor prognosis and increased metastasis (11-14). The second aim of this study was to investigate the effectiveness of PEITC as a dietary chemopreventive agent, and to determine its effect(s) on AldeFluor expressing 'stem' cells and on biomarker expression.

We investigated the effectiveness of this compound by supplementing the diet of the PyMT mice with PEITC and examining its effects macroscopically as well as microscopically for mammary disease progression. Microscopic evaluation was complemented by immunohistochemical examination of serial mammary tissues for the ER $\alpha$, FOXA1, and GATA-3 transcription factor network. In addition, in vitro studies with a mammary tumor cell line derived from these animals were performed to investigate whether PEITC targets cancer cells with 'stem/progenitor cell-like' properties using the AldeFluor assay.

\section{Materials and methods}

Mice and histology. All procedures involving mice were conducted in accordance with the National Institutes of Health regulations concerning the use and care of experimental animals. Approval from the Institutional Animal Care and Use Committee was obtained before initiating the study. Male PyMT mice of FVB/N strain were obtained from the
National Cancer Institute mouse repository and were randomly bred with wild-type FVB/N (WT) females lacking the PyMT transgene to obtain female mice heterozygous for the PyMT transgene. All of the mice analyzed in this study were female PyMT heterozygote.

Female mice were divided into treatment and control groups after weaning at 21 days. PEITC fortified or control diet was started the day after weaning. The treatment feed was made up of regular mouse feed treated with $8 \mathrm{mmol} / \mathrm{kg}$ of PEITC (Sigma, St. Louis, MO) dissolved in corn oil (Sigma), a dose that has been previously used in chemoprevention models (15). The control group received feed with the addition of plain corn oil. Based on duration of exposure to the supplemented feed prior to harvest, mice from each group were further divided into four subgroups: an indefinite or $>8$ weeks exposure ( 9 controls; 10 PEITC fed), eight weeks (8 controls; 6 PEITC fed), six weeks ( 7 controls; 5 PEITC fed), and four weeks (5 controls; 4 PEITC fed). All of the mice in the 'indefinite' group required euthanasia prior to the completion of the 16 weeks for humane reasons. These reasons included: ulceration, respiratory distress, and inhibited activity due to size of tumors such that the mouse could not eat or drink.

In order to rule out any mammary tumor formation before supplementation, all mice were adequately palpated for mammary glands and/or tumors. Bilateral abdominal mammary glands were used for whole mount preparation in addition to distinctly palpable tumors. One or more tumors from the same animal were analyzed. Mammary glands were fixed in $10 \%$ formalin, and subsequently paraffin-embedded, sectioned, and stained with hematoxylin and eosin (H\&E). Additionally, both lungs were also subjected to histological examination.

Cells and cell culture. Tumor sample was surgically isolated from a 14-week old PyMT female mouse, digested with collagenase/hyluranidase, and a cell line was established in vitro. Tumor cells were subsequently maintained in Dulbecco's Modification of Eagle's Medium (DMEM) with phenol red supplemented with $10 \%$ fetal bovine serum and penicillin/streptomycin.

Flow cytometry to measure stem-cell-like activity using AldeFluor assay. Cultured PyMT cells were treated for 24 and $48 \mathrm{~h}$ with PEITC concentrations at $5 \mathrm{nM}$ and $10 \mathrm{nM}$. ALDH1 positivity was measured using AldeFluor assay as per instructions from the manufacturer (Stem Cell Technologies, Vancouver, Canada). The assay was performed in two parts; with and without a competitive inhibitor diethylaminobenzaldehyde (DEAB).

Immunohistochemistry. To identify cells expressing $\mathrm{ER} \alpha$, FOXA1, and GATA-3, formalin-fixed mammary gland sections were immunostained using antibodies against ER $\alpha$, FOXA1, and GATA-3 (Santa Cruz Biotechnology, Santa Cruz, CA) as described previously (2). Expression was recorded in two parts; percentage of staining that ranged from ' 0 ' in case of no nuclear expression to a maximum of ' 100 ' with all cells showing expression; and intensity of $0,1+, 2+$ and $3+$ for none, weak, moderate and strong staining respectively. Percentage $(\mathrm{P})$ and intensity (I) of nuclear 


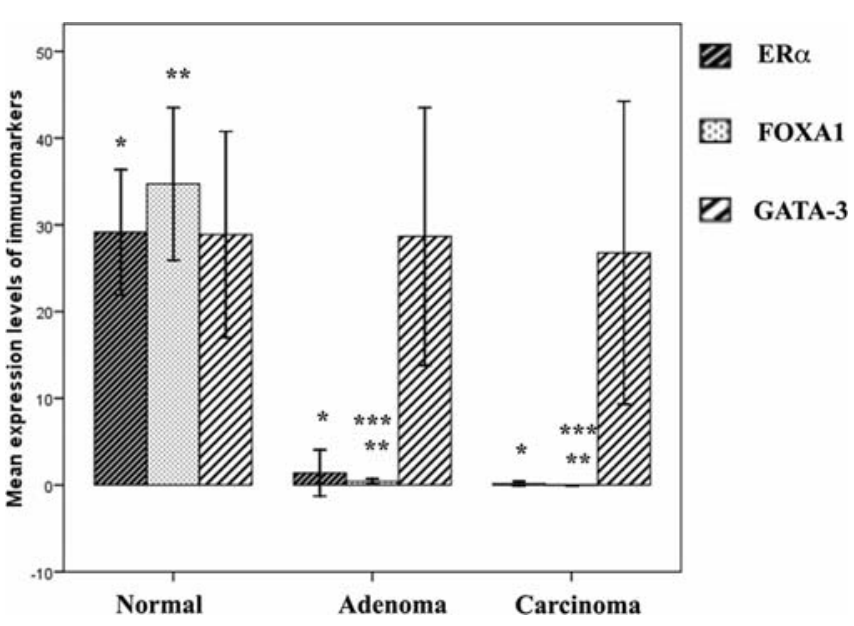

Figure 1. Graph (mean $\pm \mathrm{SE}$ ) demonstrating sequential loss of ER $\alpha$, FOXA1 and GATA-3 in various stages of tumor progression in PyMT transgenic mouse model. Although, the expression levels of ER $\alpha$ and FOXA1 are negligible in late stages, we observed a persistence of GATA-3 expression even in late stages of tumors. ${ }^{*}, *$ normal vs. adenoma or carcinoma for ER $\alpha$ and FOXA1 $\mathrm{p}<0.0001$. ${ }^{* * *}$ FOXA1, adenoma vs. carcinoma $\mathrm{p}=0.034$. Differences in GATA-3 expression were not significantly different $(\mathrm{p}=0.157)$.

expression for ER $\alpha$, FOXA1, and GATA3 were multiplied to generate a numerical expression score $(\mathrm{S}=\mathrm{P} \times \mathrm{I})(2)$.

Statistical analysis. Data from animal studies as well as immunohistochemistry were analyzed using Statistical Package for Social Sciences 17.0 (SPSS, Inc., Chicago, IL, USA). All continuous data were compared across the control and PEITC-supplemented groups using the Wilcoxon Signed Rank test. Survival curves were plotted using the KaplanMeier function. All tests were two-sided and a $\mathrm{p}=0.05$ was considered as significant.

\section{Results}

PyMT transgenic mice. Mammary tissues from mice 4 weeks of age and those that demonstrated the four distinct mammary gland lesions, hyperplasia, adenoma, low grade carcinoma and high grade carcinoma, in PyMT as described by Lin et al (6) were analyzed for the expression of biomarkers.

Expression of only GATA-3 persists in tumors of PyMT mice. Expression of ER $\alpha$, FOXA1, and GATA- 3 in breast cancer is associated with a well-differentiated phenotype as well as a favorable prognosis (2). In particular, GATA-3 is required for differentiation of luminal progenitor cells $(16,17)$. The expression pattern of ER $\alpha$ in the PyMT model has previously been reported (6), which states that ER positivity decreases in tumors as they transform to the carcinomatous stage. However, the mechanisms associated with these changes have not been well studied. Also, there are no reports examining expression patterns of GATA-3 and FOXA1 in this model. To address this, we performed immunohistochemical examination of mammary tumors at different time points. When ER $\alpha$ and FOXA1 expression was compared across normal luminal cells, adenomas, and carcinomas, there was a significant loss in their expression as lesions progressed from normal tissue
A

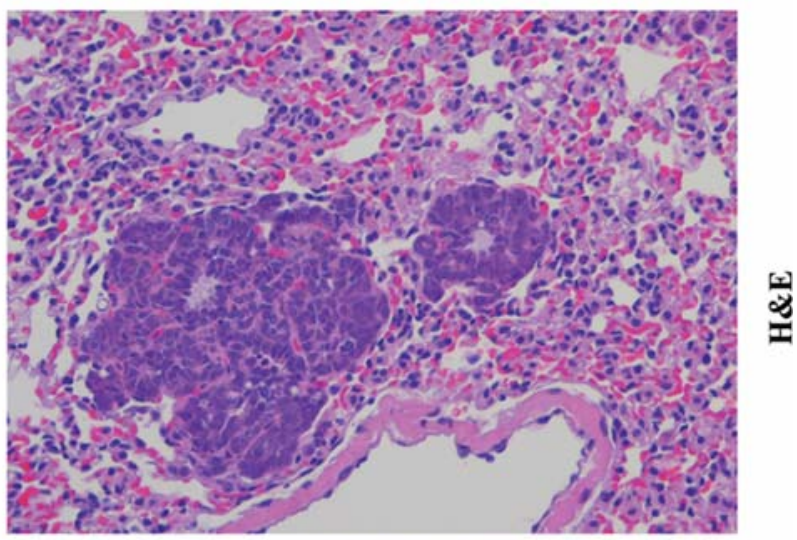

B

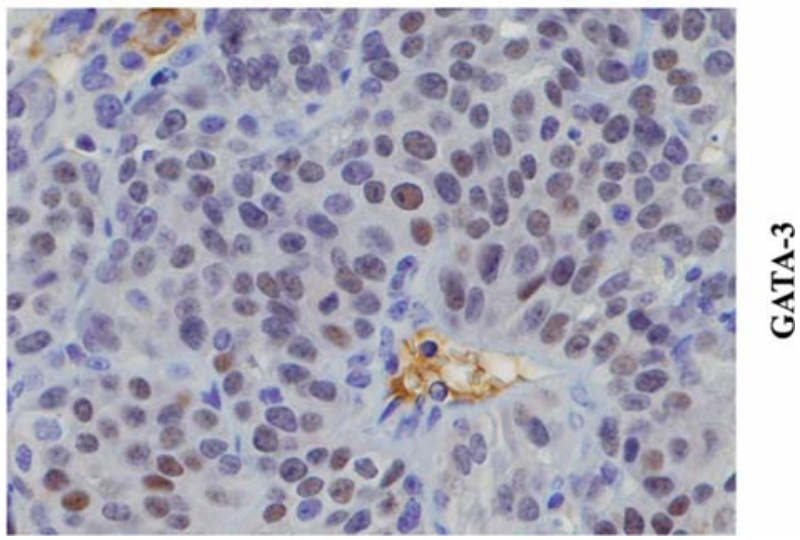

Figure 2. GATA-3 expression in lung metastatic lesions in PyMT mouse model. (A) H\&E staining of lung metastasis (magnification x20). (B) GATA-3 staining in lung metastasis (magnification $\mathrm{x} 40$ ). Note a subpopulation of cancer cells was GATA-3-positive.

to tumors ( $\mathrm{p}<0.0001$ for both markers) (Fig. 1). ER $\alpha$ expression was low in adenomas and they decreased further in carcinomas. Low FOXA1 expression was noted in adenomas but they were completely lost in carcinomas $(\mathrm{p}=0.034)$. Thus, it appears that FOXA1 expression parallels ER $\alpha$ in this model and its expression is lost. Alternatively, it is possible that FOXA1negative tumor cells are clonally selected during progression of the tumor. GATA-3 expression was much higher than that of ER $\alpha$ and FOXA1 in adenomas and carcinoma stage and did not differ significantly from the normal mammary gland $(\mathrm{p}=0.157)$. This finding demonstrates that GATA-3 persists even in late stages of tumors in this mouse model (Fig. 1).

GATA-3 expression is preserved in metastasis. Previous studies that utilized transplantation of PyMT tumor cells into syngenic mice with or without additional manipulation of GATA-3 expression in tumor cells concluded that tumor progression involves expansion of GATA-3-negative tumor cells (12). These authors concluded that GATA-3 actively induces differentiation and represses dissemination of tumor cells. As per this model, lung metastasis should lack GATA-3 expression. To test this possibility, we performed immunohistochemistry of lung tissue for metastases. In all mice with pulmonary metastases that were analyzed, we noted persistence of GATA-3 expression in a subpopulation of metastatic cells (Fig. 2). The differences observed in the 


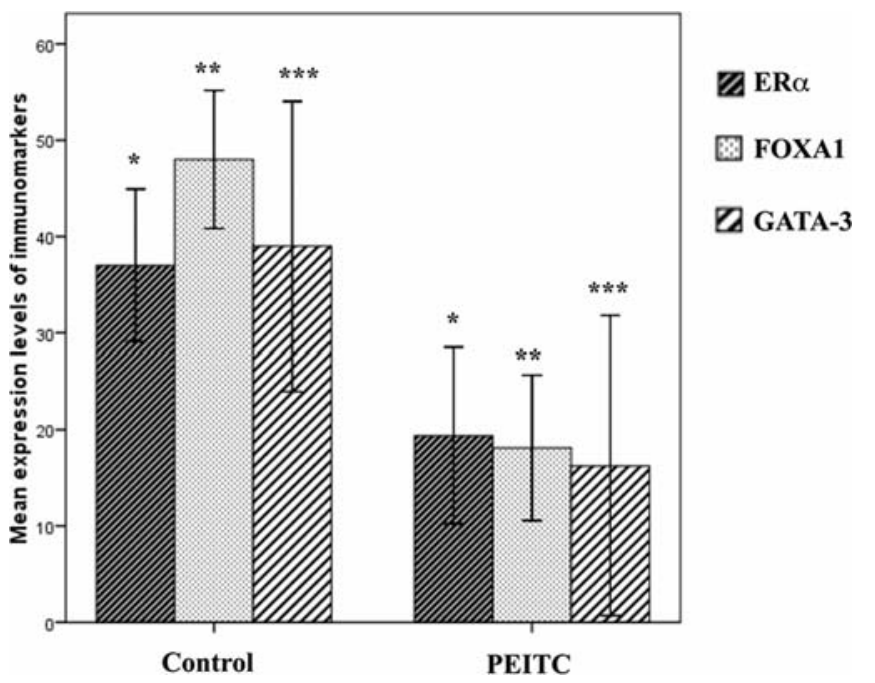

Figure 3. ER $\alpha$, FOXA1 and GATA-3 expression in normal luminal cells in both control and PEITC supplemented mice (mean \pm SE). The expression of these biomarkers in controls is 2-3 times as much as in PEITC group. Control vs. PEITC treated groups ${ }^{*} \mathrm{p}=0.001,{ }^{* *} \mathrm{p}<0.0001$, and ${ }^{* * *} \mathrm{p}=0.043$.
PEITC delays growth of tumors. In order to study the effect of a chemopreventive agent in this model, we supplemented one group of mice with PEITC. In both groups, the mean time to appearance of tumors from weaning was about 57 days. When mice supplemented with PEITC and control diets for the same duration were compared, mice from the PEITC group developed twice as many tumors as the control group. This was in part due to the small size of tumors and the fact that they did not merge to form larger tumor nodules in the PEITC group. The overall grade of tumors developed in the two supplemented groups did not vary significantly. The late tumors showed significant cytological atypia, mitotic activity, and necrosis. In addition, squamous metaplasia was also noted in many tumors. The number of mice from both groups that developed lung metastases and the number of metastatic foci did not differ significantly. In addition, we did not note any difference in the survival of mice in the indefinite group by supplementation group.

PEITC delays tumor initiation. We then examined the effects of PEITC treatment on the expression pattern of above markers on normal mammary gland. Both control and PEITCsupplemented animals showed well-formed mammary glands at 4 weeks. Although the normal luminal cells expressed ER $\alpha$,
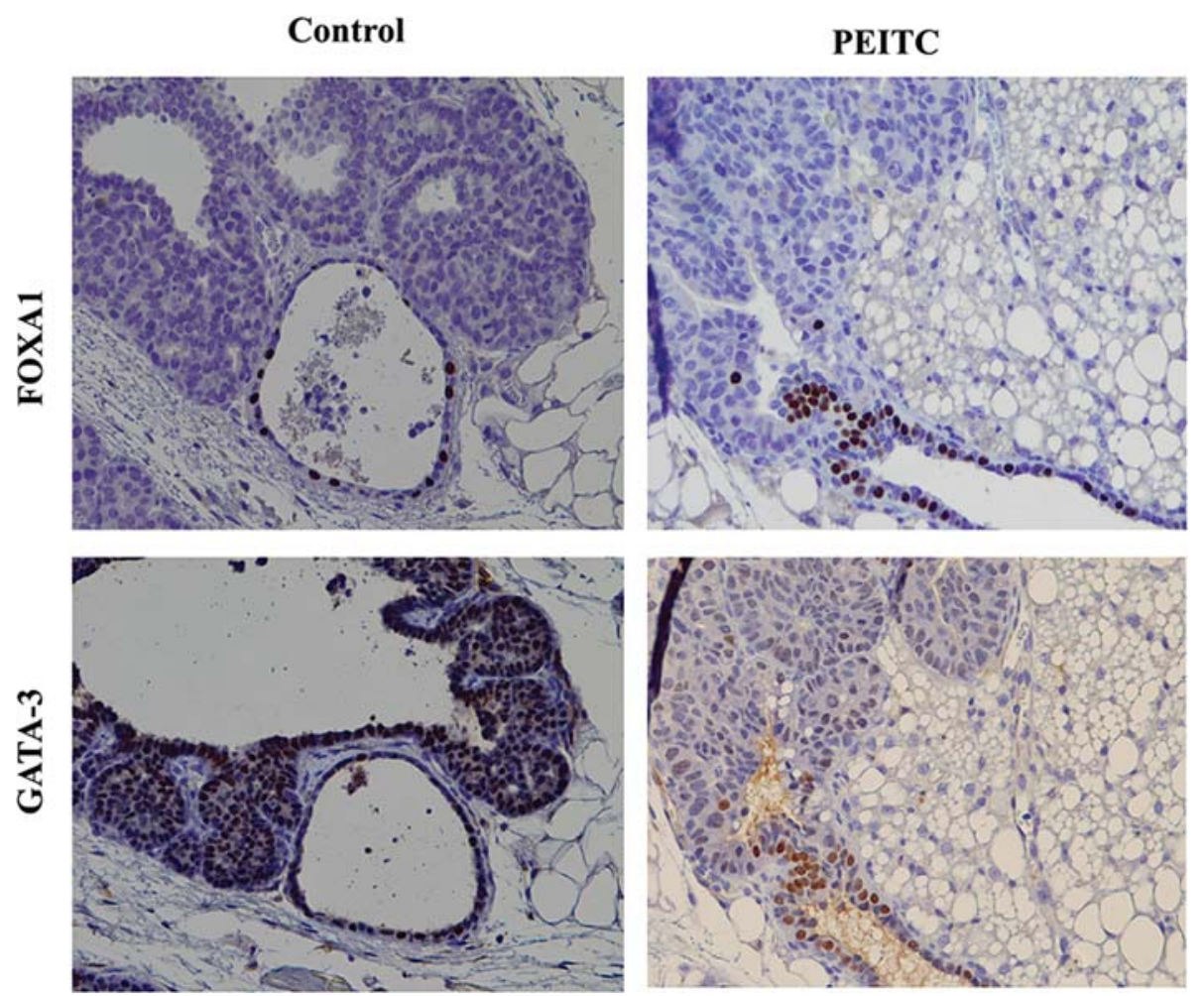

Figure 4. A representative FOXA1 and GATA-3 staining of mammary gland of untreated and PEITC treated animals maintained for four weeks after weaning (magnification $\mathrm{x} 20$ ).

previous study could be due to variations in the methods used for inducing metastasis. Unlike GATA-3, FOXA1 expression was not observed in lung metastatic cells (Fig. 2).
FOXA1 and GATA-3 in both groups, their expression was twoto three-fold higher in the control group $(\mathrm{p}=0.001,<0.0001$, and 0.043 , respectively) (Fig. 3). These differences were more 
ALDEFLUOR
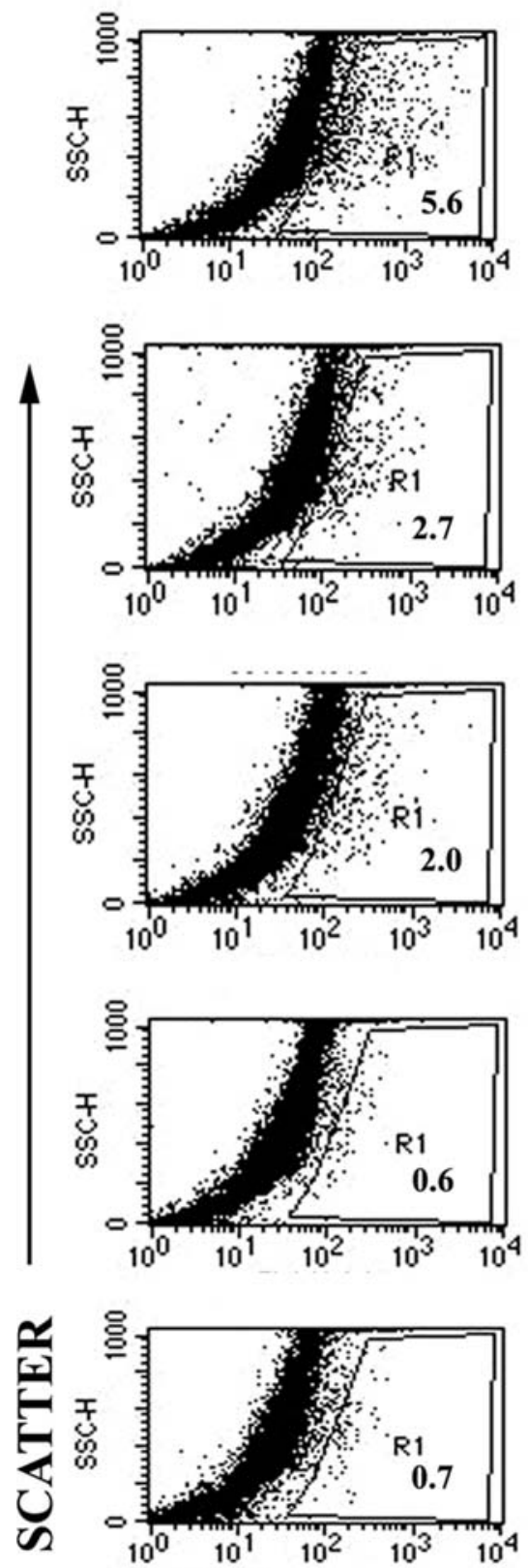

ALDH1

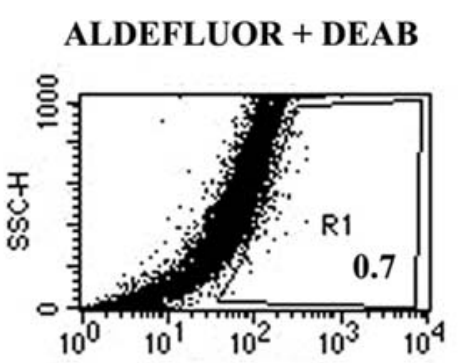

Control

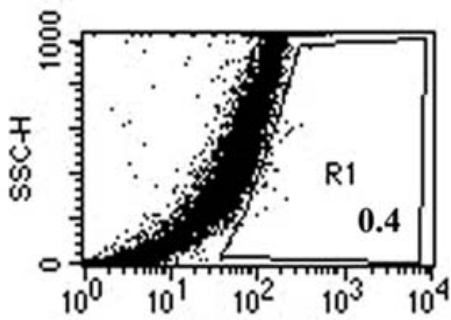

PEITC $5 \mathrm{nM}$

24 hour

PEITC 5 nM

48 hour

\section{PEITC 10 nM}

24 hour

PEITC 10 nM

48 hour

Figure 5. PEITC depletes AldeFluor-positive cancer cells. Mammary tumor cell line derived from PyMT mice was treated with PEITC for indicated time and examined for AldeFluor-positive cells by flow cytometry. Cells were incubated with fluorescent ALDH1 substrate AldeFluor with or without competitive inhibitor DEAB. R1 corresponds to AldeFluor-positive cells.

significant in mice at early age. These results suggest that PEITC inhibits initiation of these tumors, possibly by reducing the proliferation of luminal cells.

PEITC did not have an effect on the expression pattern of $\mathrm{ER} \alpha, \mathrm{FOXA1}$, and GATA-3 in tumors. This could be due to very low expression levels of these markers and any changes as a consequence of PEITC treatment were within the range of standard deviation. Representative FOXA1 and GATA-3 staining patterns in control and 4-week PEITC-treated groups are shown in Fig. 4.

PEITC targets AldeFluor-positive cells. The chemopreventive activity of PEITC involves generation of reactive oxygen species (ROS) (9). Therefore, cancer cells with effective ROS generating system are expected to be sensitive to PEITC. 'Cancer stem cells' have been shown to be efficient in scavenging reactive oxygen species, which enables them to be radioresistant (18). If PEITC is capable of inducing ROS in differentiated cancer cells but not in cancer stem cells, prolonged treatment with PEITC is expected to cause enrichment of cancer stem cells. To test this possibility, we examined the 'cancer stem cell' status of untreated and PEITC-treated PyMT mammary tumor derived cell line. Normal and cancer epithelial cells from mouse mammary gland as well as the human breast that express higher levels of aldehyde dehydrogenase 1 (ALDH1) are considered to 
have stem/progenitor cell-like properties $(19,20)$. However, cancer stem/progenitor cell phenotype of PyMT-derived tumors have not been examined so far. ALDH1 levels are usually measured using the dye AldeFluor, which provides indirect measurement of all ALDHs in cells. We examined the effect of PEITC on the AldeFluor positivity of tumor cells. As seen in Fig. 5, approximately 5\% of control cells were AldeFluor-positive compared to at least $2 \%$ positivity in PEITC-treated cells. With increasing duration and/or concentration of exposure to PEITC, tumor cells demonstrated reduced AldeFluor positivity. These results suggest that cancer cells with 'stem cell-like' properties as characterized by AldeFluor assay are sensitive to PEITC.

\section{Discussion}

The PyMT transgenic mouse has been previously demonstrated to model human breast carcinogenesis. The main advantages of this model are that it has high incidence of lung metastasis with shorter latency periods for tumor development and not being dependent on animals getting pregnant. More importantly, it is one of the few models of ER $\alpha$-positive tumors, with early stage tumors being of histological low grade and expressing both ER $\alpha$ and PR. The luminal A status of these tumors has been confirmed by gene expression studies (21). Consistent with this, we found the expression of FOXA1 and GATA-3 in these tumors.

Animals with mammary-specific PyMT transgene show a range of histological lesions depending on their age. Similar to the observations by Lin et al, we noted four distinct stages of tumor progression from hyperplasia to adenoma through low grade and high grade carcinoma (6). The late stages are associated with marked cytological atypia and mitotic activity. In addition, we observed prominent necrosis and foci of squamous metaplasia in these late stages. These morphological changes in the tumor characteristics were also associated with change in the immunophenotype. Similar to Lin et al (6), late stage tumors in this study showed marked decrease in ER $\alpha$. We additionally demonstrate a complete loss of FOXA1 expression with progression. These morphological and immunophenotypic changes suggest that these late stage tumors no longer represent luminal A phenotype. In this context, it is worth noting that using gene expression analysis Herschkowitz et al have classified the PyMT tumors as luminal A subtype (21). Given the findings of our study, it is imperative that these studies are repeated with tumors at later stages to confirm whether they are still of luminal A phenotype. In human breast cancers, it is believed that progression of tumor grade does not occur. However, Natrajan et al recently documented $16 \mathrm{q}$ deletion, characteristic of low grade cancers, in some high grade ER $\alpha$-positive tumors (22). They suggest that in at least some cases of ER $\alpha$ positive tumors progression does occur. A detailed analysis of the PyMT tumors, at different stages of development, might help to understand some of the changes associated with conversion of luminal A to luminal B phenotype.

GATA-3 is required for differentiation of luminal progenitor cells $(16,17)$. Targeted deletion of this gene in the mammary gland leads to accumulation of luminal progenitor cells and concomitant block in differentiation (16). However, survival of differentiated cells also requires GATA-3 (12). GATA-3 is suggested to function as a coregulator of ER $\alpha$ function. We noted that GATA-3 expression was higher than that of ER $\alpha$ and FOXA1 to begin with and persisted even in the late stages of tumors through metastases. In the hierarchy of luminal cell differentiation, GATA-3 appears to be upstream of FOXA1 and ER $\alpha$ because a substantially larger number of luminal cells express GATA-3 compared to ER $\alpha$ or FOXA1 both in human breast (23) and in the mouse mammary gland (Fig. 3). Therefore, differentiated luminal cells expressing GATA-3 but lacking ER $\alpha$ and FOXA1 must exist. The high expression of GATA-3 is in concordance to a previous study that used mouse model's intrinsic gene set cluster analysis to demonstrate that GATA-3 gene was highly expressed in MMTV-PyMT tumors similar to that observed in human luminal tumors (21).

Dietary prooxidant molecules such as PEITC are believed to cause apoptosis of transformed but not normal cells (7). In vitro studies have identified multiple cellular targets of PEITC: inhibition of cap-dependent translation by regulating the level and phosphorylation of $4 \mathrm{E}-\mathrm{BP} 1$, proteosomal degradation of cell division cycle $25 \mathrm{C}$ and cyclin-dependent kinase 1 leading to $\mathrm{G} 2 / \mathrm{M}$ arrest, and covalent binding to tubulin, which may be essential for growth inhibition (24-26). In this study, we have noted an effect of PEITC in early stage but not late stage tumors with respect to expression of biomarkers. The novel finding in this study is that PEITC lowers the expression of biomarkers such as ER $\alpha$, FOXA1, and GATA-3 in normal luminal cells as compared to those in control mice. Thus, even in the early stage when the ducts show some expression of these biomarkers, PEITC inhibits the initiation of tumor formation by reducing the proliferation of luminal cells that may be prone for tumorigenesis.

Although PEITC delayed tumor initiation, it did not have an effect on overall survival or time to development of tumors. One reason may be that while PEITC reduced tumor initiation

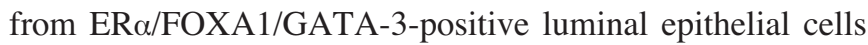
by suppressing their expression and thus the hormonal network, it may have inadvertently promoted tumor initiation/ progression by ER $\alpha$-negative luminal cells. It is more likely that PyMT is a very potent oncogene, and chemopreventive agents can inhibit only some of its activity. However, PEITC appears to arrest the tumor progression at some stage after tumor formation, possibly only a specific subtype, although this was not evident in the immunohistochemical examination of the biomarkers that we studied. Further studies on more reliable biomarkers that help differentiate the various stages of mammary gland tumorigenesis are warranted.

In vitro study of mammary tumor cells exposed to various durations and concentrations of PEITC for AldeFluor positivity determined that PEITC reduces cells with 'stem celllike' features. This suggests that stem cells may be sensitive to PEITC, if 'stem cells' are defined as AldeFluor-positive. Once further studies confirm the chemopreventive role of PEITC in breast tumorigenesis, radioresistant cancers due to 'stem cell-like' properties could be treated with PEITC (27).

In summary, the PyMT model may be ideal to study the progression of luminal A to luminal B subtype cancer and to identify genetic/epigenetic changes associated with such a 
progression. PIETC at least partially inhibits tumor initiation and delays progression in this model.

\section{Acknowledgements}

We thank Poornima Bhat-Nakshatri and Susan Rice for assistance in AldeFluor assay. This study is supported by the grant R03 CA123561 from the National Cancer Institute to HN. KM is supported by T32 cancer biology training grant CA111198 from National Institutes of Health. HN is Marian J. Morrison Investigator of Breast Cancer Research.

\section{References}

1. Sorlie T, Perou CM, Tibshirani R, et al: Gene expression patterns of breast carcinomas distinguish tumor subclasses with clinical implications. Proc Natl Acad Sci USA 98: 10869-10874, 2001.

2. Badve S, Turbin D, Thorat MA, et al: FOXA1 expression in breast cancer correlation with luminal subtype A and survival. Clin Cancer Res 13: 4415-4421, 2007.

3. Eeckhoute J, Keeton EK, Lupien M, Krum SA, Carroll JS and Brown M: Positive cross-regulatory loop ties GATA-3 to estrogen receptor alpha expression in breast cancer. Cancer Res 67: 6477-6483, 2007.

4. Cheang MC, Chia SK, Voduc D, et al: Ki67 index, HER2 status, and prognosis of patients with luminal B breast cancer. J Natl Cancer Inst 101: 736-750, 2009.

5. McCune K, Bhat-Nakshatri P, Thorat MA, Nephew KP, Badve S and Nakshatri H: Prognosis of hormone-dependent breast cancers: implications of the presence of dysfunctional transcriptional networks activated by insulin via the immune transcription factor T-bet. Cancer Res 70: 685-696, 2010.

6. Lin EY, Jones JG, Li P, et al: Progression to malignancy in the polyoma middle $\mathrm{T}$ oncoprotein mouse breast cancer model provides a reliable model for human diseases. Am J Pathol 163: 2113-2126, 2003.

7. Wu XJ and Hua X: Targeting ROS: selective killing of cancer cells by a cruciferous vegetable derived pro-oxidant compound. Cancer Biol Ther 6: 646-647, 2007.

8. Reen RK, Dombkowski AA, Kresty LA, et al: Effects of phenylethyl isothiocyanate on early molecular events in N-nitrosomethylbenzylamine-induced cytotoxicity in rat esophagus. Cancer Res 67: 6484-6492, 2007.

9. Trachootham D, Zhou Y, Zhang H, et al: Selective killing of oncogenically transformed cells through a ROS-mediated mechanism by beta-phenylethyl isothiocyanate. Cancer Cell 10: 241-252, 2006.

10. Thorat MA, Marchio C, Morimiya A, et al: FOXA1 expression in breast cancer is associated with Luminal subtype and good prognosis. J Clin Pathol 2007.

11. Jacquemier J, Charafe-Jauffret E, Monville F, et al: Association of GATA3, P53, Ki67 status and vascular peritumoral invasion are strongly prognostic in luminal breast cancer. Breast Cancer Res 11: R23, 2009.
12. Kouros-Mehr H, Bechis SK, Slorach EM, et al: GATA-3 links tumor differentiation and dissemination in a luminal breast cancer model. Cancer Cell 13: 141-152, 2008.

13. Mehra R, Varambally S, Ding L, et al: Identification of GATA3 as a breast cancer prognostic marker by global gene expression meta-analysis. Cancer Res 65: 11259-11264, 2005.

14. Dydensborg AB, Rose AA, Wilson BJ, et al: GATA3 inhibits breast cancer growth and pulmonary breast cancer metastasis. Oncogene 28: 2634-2642, 2009.

15. Conaway CC, Wang CX, Pittman B, et al: Phenethyl isothiocyanate and sulforaphane and their $\mathrm{N}$-acetylcysteine conjugates inhibit malignant progression of lung adenomas induced by tobacco carcinogens in A/J mice. Cancer Res 65: 8548-8557, 2005.

16. Asselin-Labat ML, Sutherland KD, Barker H, et al: Gata-3 is an essential regulator of mammary-gland morphogenesis and luminal-cell differentiation. Nat Cell Biol 9: 201-209, 2007.

17. Kouros-Mehr H, Slorach EM, Sternlicht MD and Werb Z: GATA-3 maintains the differentiation of the luminal cell fate in the mammary gland. Cell 127: 1041-1055, 2006.

18. Diehn M, Cho RW, Lobo NA, et al: Association of reactive oxygen species levels and radioresistance in cancer stem cells. Nature 458: 780-783, 2009.

19. Ibarra I, Erlich Y, Muthuswamy SK, Sachidanandam R and Hannon GJ: A role for microRNAs in maintenance of mouse mammary epithelial progenitor cells. Genes Dev 21: 3238-3243, 2007.

20. Ginestier C, Hur MH, Charafe-Jauffret E, et al: ALDH1 is a marker of normal and malignant human mammary stem cells and a predictor of poor clinical outcome. Cell Stem Cell 1: 555-567, 2007.

21. Herschkowitz JI, Simin K, Weigman VJ, et al: Identification of conserved gene expression features between murine mammary carcinoma models and human breast tumors. Genome Biol 8: R76, 2007.

22. Natrajan R, Lambros MB, Geyer FC, et al: Loss of 16q in high grade breast cancer is associated with estrogen receptor status: Evidence for progression in tumors with a luminal phenotype? Genes Chromosomes Cancer 48: 351-365, 2009.

23. Nakshatri $H$ and Badve S: FOXA1 in breast cancer. Expert Rev Mol Med 11: E8, 2009.

24. Hu J, Straub J, Xiao D, et al: Phenethyl isothiocyanate, a cancer chemopreventive constituent of cruciferous vegetables, inhibits cap-dependent translation by regulating the level and phosphorylation of 4E-BP1. Cancer Res 67: 3569-3573, 2007.

25. Xiao D, Johnson CS, Trump DL and Singh SV: Proteasomemediated degradation of cell division cycle $25 \mathrm{C}$ and cyclindependent kinase 1 in phenethyl isothiocyanate-induced G2-Mphase cell cycle arrest in PC-3 human prostate cancer cells. Mol Cancer Ther 3: 567-575, 2004.

26. Mi L, Xiao Z, Hood BL, et al: Covalent binding to tubulin by isothiocyanates. A mechanism of cell growth arrest and apoptosis. J Biol Chem 283: 22136-22146, 2008.

27. Phillips TM, McBride WH and Pajonk F: The response of CD24(-/low)/CD44+ breast cancer-initiating cells to radiation. J Natl Cancer Inst 98: 1777-1785, 2006. 